\title{
Research article \\ Evaluation of serum macronutrients level and depression among adolescents and its association with pre-hypertension: A cross sectional study
}

\author{
Abeetha S. ${ }^{1}$, Thirunavukarasu R. ${ }^{2}$, Divya R. ${ }^{3}$, Suma S. ${ }^{4}$, Ganesh M. ${ }^{5}$, Sadhana S. ${ }^{6}$, Mugunthan ${ }^{7}$ \\ ${ }^{1}$ Associate Professor, ${ }^{4}$ Assistant Professor, ${ }^{5}$ Professor and Head, Department of Physiology, ${ }^{7}$ III Year MBBS student, ACS \\ Medical College and Hospital, Chennai, Tamil Nadu. India \\ ${ }^{2}$ Associate Professor, Department of Community Medicine, Pudukkottai Government Medical College, Pudukkottai, Tamil \\ Nadu, India \\ ${ }^{3}$ Associate Professor, Department of Physiology, Dhanalakshmi Srinivasan Medical College and Hospital, Perambalur, Tamil \\ Nadu, India \\ ${ }^{6}$ Scientist B, National institute of nutrition, Hyderabad, Telangana, India
}

(Received: January 2021 Revised: August $2021 \quad$ Accepted: September 2021)

Corresponding author: Suma S. Email: msrenu01@gmail.com

\begin{abstract}
Introduction and Aim: Serum macronutrients measurement prove to be precedent values with regard to diagnose hypertension at an earlier age. Depression in any form could be ruinous to younger generation, also could precipitate hypertension. The aim of the study is to estimate serum calcium, magnesium levels, grade depression and to establish its association with prehypertension.

Materials and Methods: A cross sectional study was conducted on 68 students aged 17-19 years over a two months period. Blood samples were drawn and serum magnesium and calcium were estimated. Depression was assessed by administering KADS - 6 questionnaires. Blood Pressure was measured by auscultatory method using mercury sphygmomanometer. All parameters were presented as mean $\pm \mathrm{SD}$. Chi square test was used to compare proportions for the groups. Unpaired t test was used to compare the groups. ANOVA was used to compare the groups for continuous variables. Statistical analysis was done using Epi info (version 6.04d) software package.

Results: More than $44 \%$ of the study participants had elevated serum calcium levels, while about $10 \%$ of them had elevated serum magnesium levels. More than half (58.8\%) of the participants were pre-hypertensive. One third of the study participants were depressed of which about $10 \%$ of them had severe depression. The prevalence of prehypertension was 2.1 times higher among participants having hypercalcemia when compared to participants who have normal serum calcium levels with $95 \%$ CI of $1.38-3.22$ which is statistically significant (p-value < 0.001). The study could not establish a statistical difference between different groups with regards to serum magnesium levels and depression.
\end{abstract}

Conclusion: Our study portrays the widely prevalent depression and pre-hypertension in younger generation and exhibits firm evidence on measuring serum macronutrient levels to diagnose pre-hypertension before time that can prevent the disease load and help ineffective management.

Keywords: Serum macronutrients; depression; pre-hypertension.

\section{INTRODUCTION}

$\mathrm{H}$ ypertension is one of the most common non communicable diseases that affect both males and females almost in equal ratio. It is a known fact that hypertension in early ages can worsen with age that incredibly increases morbidity and mortality. Majority of the Indian studies on hypertension is done among adult population which gives an impression that it is a disease affecting the middle aged. But the adolescent prehypertension is underreported. Lack of space and time in the recent times has restricted the adolescents to engage themselves in indoor activities. Also, the current life style of the younger generation is more towards predisposition to non-communicable diseases like obesity, depression and hypertension.

There are studies showing increased incidence of cardiovascular diseases among the population with decreased dietary intake of potassium, magnesium, and calcium. In particular, serum magnesium has a significant inverse correlation with incidence of cardiovascular diseases (1). Magnesium, the orphan ion, regulates blood pressure levels by modulating vascular tone and also maintains smooth conduction of cardiac impulses with proven antiarrhythmic effect. Fluctuation in extracellular magnesium levels affect calcium concentration thereby altering arterial smooth muscle tone by release of Nitric oxide. Hence, it has been suggested that magnesium deficiency is related to the pathophysiology of hypertension and atherosclerosis (2).

Hypertension is caused due to a disruption in calcium homeostasis. Calcium plays a vital role as second messenger in vascular smooth muscle excitationcontraction coupling. The increased intracellular calcium level contributes to increased peripheral 
vascular resistance (PVR) leading to hypertension (3).

Low serum phosphate has been linked with the development of hypertension as it increases the sympathoadrenal activity in hypertensive patients, as epinephrine leads to a net shift of phosphate from the extracellular to the intracellular compartment. Moreover, serum phosphate level is inversely related to BP in normotensive individuals (4). Obesity is recognized as a major risk factor for the development of hypertension. Obesity-associated hypertension causes an inadequate vasodilatation in the presence of increased blood volume and cardiac output, which is a consequence of an increased body mass. Obesity is one of the significant risk factors which alters peripheral vascular resistance that contributes to diastolic blood pressure. Systolic blood pressure range is also determined by changes in diastolic blood pressure (5).

Currently the prevalence of depression is $4.8-8.6 \%$. In a span of another twenty years depressive illness could be the second major leading cause of disability. Hypertension and depression could be two interlinked disorders as an increased sympathetic tone is observed in both the conditions. There is also an increase in the levels of ACTH and cortisol among patients with depression and hypertension $(6,7)$.

As prevention is always better than cure, research in this area will help us in early detection of prehypertension that will aid in effective management of hypertension. The objectives of the study were to estimate the serum macro nutrient levels among the study subjects, to assess depression score among the study population and to measure blood pressure among study participants and to find association with above findings.

\section{METHODOLOGY}

\section{Study design}

A cross sectional study of two months' duration with a sample size of 68 was taken. Simple random sampling technique was used.

\section{Inclusion criteria}

The present study includes adolescents between 17 and 19 years.

\section{Exclusion criteria}

The present study excludes subjects with any diagnosed non-communicable disease (Diabetes Mellitus, Hypertension, Dyslipidemia, Endocrine disorders, renal disease, and cardiovascular disease) and respondents with any blood dyscrasias.

\section{Ethical issues}

Ethical principles such as respect for the persons, beneficence and justice was adhered. Clearance from the Institutional Review Board was obtained prior before conducting the study. Blood samples collected for the study purpose were drawn under strict aseptic precautions by the investigator after obtaining an informed consent. S/he will have the right to decide about participating or leaving the study. No information was withheld from the respondent.

\section{Equipment and other related facilities}

The study required equipment like Sphygmomanometer, Stethoscope. Blood samples were drawn and analyzed Cobas Miras Plus Automated Chemistry Analyser (USA) at the college laboratory after obtaining prior permissions. Reagent kits were supplied by AGAPPE diagnostics, Kerala, India.

\section{Data collection}

Data collection was done, after obtaining an informed consent. A questionnaire was administered to collect the data. The questionnaire covered information on identity of the subject, family history and medical history. A self- directed questionnaire was administered to assess levels of depression. Blood sample was collected to measure serum macronutrient levels (Magnesium and Calcium).

\section{Blood pressure}

Blood pressure was measured three times by the principal investigator with a time interval of two minutes between each recording. After giving five minutes rest to the participant in the departmental laboratory, blood pressure was measured in sitting posture by mercury sphygmomanometer. The pressure at which Korotkoff's sound first heard (Phase I) was taken as systolic blood pressure and the pressure at which these sounds disappeared (Phase V) was taken as diastolic blood pressure. The average of three readings was taken as appropriate systolic and diastolic blood pressure (8-10).

\section{Biochemical parameters}

Three $\mathrm{ml}$ of whole blood was collected from the study population with dry disposable syringe and needle by venous puncture under all aseptic precautions. Then serum was separated after subjecting the collected blood to centrifugation at $2500 \mathrm{rpm}$ for five minutes. Serum will be divided into two aliquots. One aliquot will be used for estimation of serum magnesium and other one for calcium.

\section{Depression scale}

6- Item Kutcher Adolescent Depression Scale (KADS - 6) was given to the study participants which has six close ended questions to be answered. Based on the scoring the study participants were grouped to be probably depressed or not depressed. 


\section{Analysis of quantitative and qualitative data}

The data was entered and analysed by using Epi info (version 6.04d) software package. All parameters were presented as mean \pm standard deviation (mean \pm $\mathrm{SD})$. Chi square test was used to compare proportions for the groups. Unpaired t test was used to compare the groups. ANOVA was used to compare the groups for continuous variables.

\section{Implication}

The study determined the prevalence of adolescent prehypertension and its associated risk factors. Also, by knowing the significance of serum macronutrient levels among pre-hypertensives, early detection and prevention of frank hypertension becomes possible just by increasing the dietary supplementation of the same.

\section{RESULTS}

Table 1: Measure of Serum Calcium, Magnesium and Blood Pressure

\begin{tabular}{|c|c|c|}
\hline \multicolumn{2}{|c|}{ Variable } & Frequency (\%) \\
\hline \multirow{2}{*}{$\begin{array}{c}\text { Serum } \\
\text { calcium }\end{array}$} & Normal & $38(55.9)$ \\
\cline { 2 - 3 } & Hypercalcemia & $30(44.1)$ \\
\hline \multirow{2}{*}{$\begin{array}{c}\text { Serum } \\
\text { Magnesium }\end{array}$} & Normal & $61(89.7)$ \\
\cline { 2 - 3 } $\begin{array}{c}\text { Blood } \\
\text { Pressure }\end{array}$ & Hypermagnesemia & $7(10.3)$ \\
\cline { 2 - 3 } & Prehypertension & $28(41.2)$ \\
\hline
\end{tabular}

Display of more than $44 \%$ of the study participants had elevated serum calcium levels, while about $10 \%$ of them had elevated serum magnesium levels. More than half $(58.8 \%)$ of the participants were prehypertensive.

Table 2: Average Serum Calcium and Magnesium levels

\begin{tabular}{|c|c|c|}
\hline Variable & Mean & SD \\
\hline Serum Calcium & 10.3 & 2.6 \\
\hline $\begin{array}{c}\text { Serum } \\
\text { Magnesium }\end{array}$ & 2.03 & 0.47 \\
\hline
\end{tabular}

Table 3: Prevalence of Depression

\begin{tabular}{|c|c|}
\hline Depression level & Frequency (\%) \\
\hline Normal & $45(66.2)$ \\
\hline Mild & $9(13.2)$ \\
\hline Moderate & $6(8.8)$ \\
\hline Severe & $2(2.9)$ \\
\hline Very Severe & $6(8.8)$ \\
\hline
\end{tabular}

One third of the study participants were depressed of which about $10 \%$ of them had severe depression.
Table 4: Association of Serum calcium, Magnesium and depression with blood pressure

\begin{tabular}{|c|c|c|c|c|}
\hline \multirow[t]{2}{*}{ Variables } & \multicolumn{2}{|c|}{$\begin{array}{c}\text { Pre- } \\
\text { hypertension }\end{array}$} & \multirow{2}{*}{$\begin{array}{l}\text { Prevalence } \\
\text { Ratio (95\% } \\
\text { CI) }\end{array}$} & \multirow[t]{2}{*}{$\mathrm{P}$ value } \\
\hline & Yes & No & & \\
\hline \multicolumn{5}{|c|}{ Serum Calcium } \\
\hline Normal & 15 & 23 & 1 & \multirow[t]{2}{*}{0.0001} \\
\hline $\begin{array}{c}\text { Hyper- } \\
\text { calcemia }\end{array}$ & 25 & 5 & $\begin{array}{c}2.1(1.38- \\
3.22)\end{array}$ & \\
\hline \multicolumn{5}{|c|}{ Serum Magnesium } \\
\hline Normal & 36 & 25 & 1 & \multirow[t]{2}{*}{0.613} \\
\hline $\begin{array}{c}\text { Hyper- } \\
\text { magnesemia }\end{array}$ & 4 & 3 & $\begin{array}{c}0.97(0.49- \\
1.90)\end{array}$ & \\
\hline \multicolumn{5}{|c|}{ Depression } \\
\hline Normal & 24 & 21 & 1 & \\
\hline Mild & 7 & 2 & $\begin{array}{c}1.46(0.94- \\
2.27)\end{array}$ & 0.200 \\
\hline Moderate & 3 & 3 & $\begin{array}{c}0.93(0.40- \\
2.19)\end{array}$ & $>0.999$ \\
\hline Severe & 2 & 0 & $\begin{array}{c}1.87(1.42- \\
2.46)\end{array}$ & 0.600 \\
\hline Very Severe & 4 & 2 & $\begin{array}{c}1.25(0.67- \\
2.34)\end{array}$ & 0.864 \\
\hline
\end{tabular}

The prevalence of prehypertension was 2.1 times higher among participants having hypercalcemia when compared to participants who have normal serum calcium levels with 95\% CI of $1.38-3.22$ which is statistically significant ( $p$-value $<0.001)$. The study could not establish a statistical difference between different groups with regards to serum magnesium levels and depression.

\section{DISCUSSION}

In present world the younger population are subjected to numerous factors including social media distractions and peer pressure from society in various forms attributing to a stressful environment. This insight warrants us to seriously take up the issue and seek out ways to formulate early detection of this stress developing to be hypertension in later years of life. Hence, we wanted to conduct a study in younger population aimed at estimating the serum macronutrients namely magnesium, calcium and assessed depression and employed above findings to find a correlation to predict hypertension at an early age.

Our study shows around 59\% subjects with prehypertension. There are enormous studies supporting our idea and have results same as ours, a few are quoted here. In a study conducted in central Delhi on 315 adolescents $1.6 \%$ were found to have systolic hypertension while $5.4 \%$ were found to have diastolic hypertension while $4.1 \%$ of the participants were systolic pre-hypertensive and $26 \%$ were diastolic prehypertensive (11). A study was conducted by Sorof $e t$ al., among obese adolescents. The results showed an increase in the prevalence of isolated systolic hypertension to be $94 \%$ (12). The results are alarming as isolated systolic hypertension is one of the major 
risk factors for cardiovascular morbidity and mortality in adults.

Literature suggests a decreased serum magnesium levels could lead to insulin resistance, hyperglycaemia, oxidative stress, endothelial dysfunction, proinflammatory state and platelet aggregation. On identifying pre-hypertensive subjects, we wanted to compare the serum macronutrients magnesium and calcium among subjects. In our study subjects with normal magnesium levels showed increased blood pressure levels compared to subjects with high magnesium levels, though the numbers are not statistically significant. Yet another study shows supportive evidence that dietary intake of magnesium and hypertension share an inverse dose-response relationship, though the evidence is limited (13). The conflicting results of studies evaluating the effects of magnesium supplements on blood pressure and other cardiovascular outcomes truly indicate that the action of magnesium in the vascular system is present but not yet established comprehensively (1).

Calcium deposits on arterial walls cause thickening and can increase peripheral resistance resulting in hypertension. An epidemiological study compared mortality rate of circulatory diseases among residents who consumed hard and soft water. Residents who consumed hard water rich in calcium and magnesium showed decreased mortality rate. Our study depicts pre-hypertension in 25 subjects with hypercalcemia. A study conducted in rural parts of China on 2023 students on childhood hypertension defined hypertension as systolic blood pressure and/or diastolic blood pressure $\geq 95$ th percentile for age and gender. According to the results of multivariable linear and logistic regression analysis, it was found that higher serum calcium levels were positively associated with childhood hypertension (15).

Familial history and psychosocial stress are important risk factors contributing to depression in adolescence. Also, genetic inheritance, developmental issues, hormonal and psychosocial interaction significantly increases the risk (16). Our study shows around 33\% of subjects having some range of depression evaluated by administering 6- Item Kutcher Adolescent Depression Scale (KADS - 6) questionnaire. About $10 \%$ of our subjects also proved to have severe depression.

16 of our subjects identified with some form of depression by KADS- 6 questionnaire fell under the pre-hypertensive range though the results were not statistically significant. In a similar study, researchers did a multi-centric cohort on young individuals, observed them for five years and established that participants who had recorded high score on Center for Epidemiological Diseases Depression scale (CESD) administered earlier during the study had significantly higher risk of developing hypertension than the counterpart with lower scores during subsequent follow-up (17).

There are certain lacunae in our study. We measured only serum calcium and magnesium whereas there are other macronutrients too which provide substantial information about the blood pressure in an individual. Obesity could be another potential predictor of prehypertension which we have failed to consider. Statistical sample size though calculated could not be adhered to. Also, we declare there are no conflicts of interest.

\section{CONCLUSION}

In our study we have tried to bring about a strong predilection of serum macronutrient levels namely calcium and magnesium as a predictor of hypertension in younger age. We did provide evidence of prevailing multifaceted depression owing to peer reviewed and pressurized social lifestyle existent among our younger generation. We also established a positive correlation between serum magnesium, calcium, and depression with prehypertension. Hence our study establishes appropriate evidence-based facts nevertheless warrants further provocative approach to diagnose pre-hypertension at an earlier age and control the devastating disease by prompt early diagnosis and emphasize lifestyle modification.

\section{ACKNOWLEDGEMENT}

I hereby acknowledge my co-authors and study participants for participating in the study.

\section{CONFLICT OF INTEREST}

Authors declare that there is no conflict of interest for the study.

\section{REFERENCES}

1. Cunha, A. R., Umbelino, B., Correia, M. L., Neves, M. F. Magnesium and vascular changes in hypertension. International journal of hypertension. 2012 Feb 29; 2012.

2. Barbagallo, M., Dominguez, L. J., Galioto, A., Ferlisi, A., Cani, C., Malfa, L., et al., Role of magnesium in insulin action, diabetes and cardio-metabolic syndrome $\mathrm{X}$. Molecular Aspects of Medicine. 2003; 24(1-3): 39-52.

3. Sudhakar, K., Sujatha, M., Babu, S. R., Padmavathi, P., Reddy, P. P. Serum calcium levels in patients with essential hypertension and their first-degree relatives. Indian $\mathrm{J}$ Clin Biochem. 2004; 19: 21-23.

4. Body, J. J., Cryer, P. E., Offord, K. P., Heath, H. $3^{\text {rd }}$. Epinephrine is a hypophosphatemic hormone in man physiological effects of circulating epinephrine on plasma calcium, magnesium phosphorus, parathyroid hormone and calcitonin. J Clin Invest. 1983; 71: 572-578.

5. Sorof J, Daniels S. Obesity and hypertension in children: A problem of epidemic proportions. Hypertension. 2002; 40: 441-447.

6. Pignone, M. P., Gaynes, B. N., Rushton, J. L., Burchell, C. M., Orleans, C. T., Mulrow, C. D., et al., Screening for depression in adults: A summary of the evidence for the 
U.S. Preventive Services Task Force. Ann Intern Med. 2002; 136: 765-776.

7. Meng, L., Chen, D., Yang, Y., Zheng, Y., Hui, R. Depression increases the risk of hypertension incidence: A meta-analysis of prospective cohort studies. J Hypertens. 2012; 30: 842-851.

8. Tambe, B., Phadke, V., Kharche, S., Joshi, R. Correlation of blood pressure with body mass index and waist to hip ratio in middle aged men. Internet Journal of Medical Update. 2010; 5(2): 26-30.

9. The seventh report of the Joint National Committee on prevention, detection, evaluation and treatment of high blood pressure. JAMA 2003; 289: 2560-2571.

10. Xing, L. Y., Diao, W. L., Liu, Y. Q., Yu, L. Y., Liu, L., Li, N., et al., Prevalence, awareness, treatment, control of hypertension in rural adult residents of Liaoning province. Zhonghua Xin Xue Guan Bing za zhi. 2010; 38(7): 652-655.

11. Anand, T., Ingle, G. K., Meena, G. S., Kishore, J., Kumar, R. Hypertension and its correlates among school adolescents in delhi. Int J Prev Med. 2014; 5(Suppl 1): S65-S70.

12. Sorof, J. M., Poffenbarger, T., Franco, K., Bernard, L., Portman, R. J. Isolated systolic hypertension, obesity, and hyperkinetic hemodynamic states in children. J Pediatr. 2002; 140: 660-666.

13. Han, H., Fang, X., Wei, X., Liu,Y., Jin, Z., Chen,Q., et al., Dose-response relationship between dietary magnesium intake, serum magnesium concentration and risk of hypertension: a systematic review and meta-analysis of prospective cohort studies. Nutr J. 2017; 16(1): 26.

14. Schroeder, H. A. Relations between hardness of water and death rates from certain chronic and degenerative diseases in the United States. J Chronic Dis 1960; 12: 586-591.

15. Sun, H., Shi, J., Wang, H., Fu, L., Zhou, B., Wu, X., et al., Association of serum calcium and hypertension among adolescents aged 12-17 years in the rural area of Northeast China. Biol Trace Elem Res. 2013 Dec; 155(3): 344-351.

16. Thapar, A., Collishaw, S., Pine, D.S., Thapar, A. K. Depression in adolescence. Lancet 2012; 379(9820): 1056-1067.

17. Davidson, K., Jonas, B. S., Dixon, K. E., Markovitz, J. H. Do Depression Symptoms Predict Early Hypertension Incidence in Young Adults in the CARDIA Study? Arch Intern Med. 2000; 160(10): 1495-1500. 